\title{
On Prime Divisors of Binomial Coefficients
}

\author{
By Pierre Goetgheluck
}

\begin{abstract}
This paper, using computational and theoretical methods, deals with prime divisors of binomial coefficients: Geometric distribution and number of distinct prime divisors are studied. We give a numerical result on a conjecture by Erdös on square divisors of binomial coefficients.
\end{abstract}

1. Introduction. In [5], a method for obtaining the expansion into primes of binomial coefficients was given. In this way, some problems about binomial coefficients can be examined using a computational approach. In this article, we give three examples:

(1) We show and explain the geometric distribution of prime divisors of binomial coefficients.

(2) We remark on the number of prime distinct divisors of binomial coefficients.

(3) New results are given about the Erdős conjecture: for $n>4,\left(\begin{array}{c}2 n \\ n\end{array}\right)$ is never squarefree.

Notation. Throughout this paper, $n, k, p, i$ are nonnegative integers satisfying $k \leq n, p$ prime. For any $x$ real, $[x]$ denotes the largest integer satisfying $[x] \leq x$. We set $d(n, k, p, i)=\left[n / p^{i}\right]-\left[k / p^{i}\right]-\left[(n-k) / p^{i}\right]$.

We recall that:

(A) The value of $d(n, k, p, i)$ is always 0 or 1 (obvious, using base $p$ ).

(B) In the expansion of $\left(\begin{array}{l}n \\ k\end{array}\right)$ into primes, the exponent of $p$ is $\sum_{i>0} d(n, k, p, i)$ (easy: write $\left(\begin{array}{l}n \\ k\end{array}\right)=n ! /(k !(n-k) !)$ and calculate the exponents of $p$ in $n !, k !$ and $(n-k) !)$.

(C) The exponent of $p$ in the expansion of $\left(\begin{array}{l}n \\ k\end{array}\right)$ into primes equals the number of borrow (s) in the subtraction $n-k$ in base $p$ (see [5]).

Result $(\mathrm{C})$ gives an easy way to get the expansion of a binomial coefficient into primes on a computer. A detailed algorithm will be found in [5].

\section{Geometric Distribution of Prime Divisors of Binomial Coefficients.}

To get an idea about the distribution of prime divisors of binomial coefficients, for a given $n$, we draw a graph with $p$ - and $k$-axes: For each $p$ (prime) and each $k$, $(p, k)$ is plotted if and only if $p$ divides $\left(\begin{array}{l}n \\ k\end{array}\right)$. Figure 1 shows the pattern for $n=600$. It is not a particular case: we get the same kind of pattern for any $n$.

Received May 27, 1987.

1980 Mathematics Subject Classification (1985 Revision). Primary 11B65, 11A51, 11 Y05. 


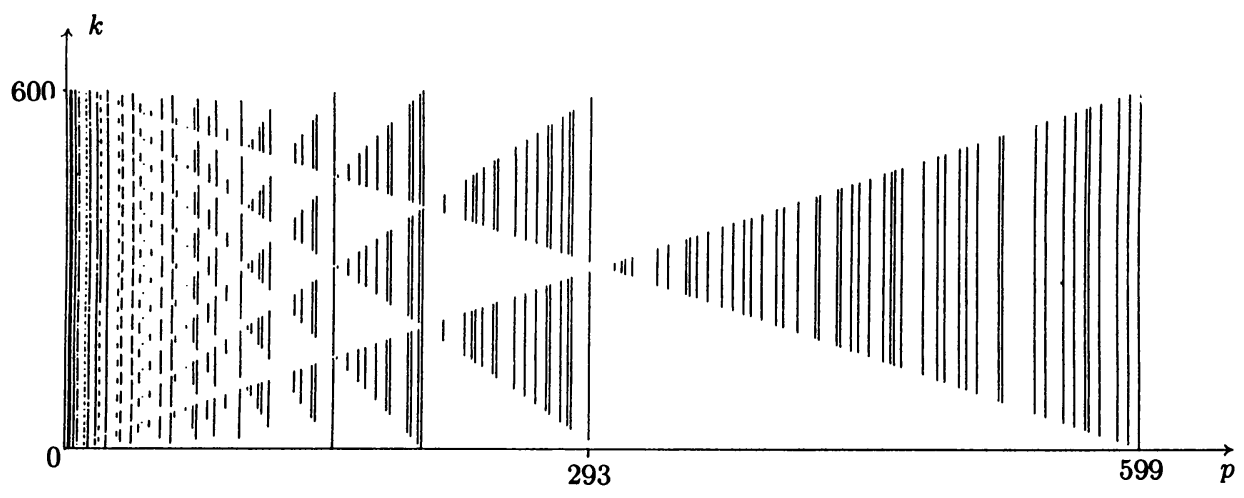

FIGURE 1

Prime divisors of $\left(\begin{array}{c}600 \\ k\end{array}\right)$.

We now explain the structure of this pattern. Let $n$ be given and $E_{i}=\{(p, k): p$ prime, $d(n, k, p, i)=1\}$. From (A) and (B), $\left(\begin{array}{l}n \\ k\end{array}\right)$ is divisible by $p$ if and only if $(p, k) \in \bigcup_{i>0} E_{i}$ and the exponent of $p$ in the expansion of $\left(\begin{array}{l}n \\ k\end{array}\right)$ into primes equals the number of $i$ 's such that $(p, k) \in E_{i}$. For a given $n$ it is easy to draw sets $E_{i}$ in $p$ - and $k$-axes. $E_{1}$ consists of triangular areas whose boundaries are

$$
\begin{aligned}
& \text { vertical lines } p=n, \quad p=n / 2, \quad p=n / 3, \ldots \\
& \text { and lines } \quad k=p, \quad k=2 p, \quad k=3 p, \ldots \text {, } \\
& k=n-p, \quad k=n-2 p, \quad k=n-3 p, \ldots .
\end{aligned}
$$

More generally, the boundaries of $E_{i}$ are

$$
\begin{aligned}
& \text { vertical lines } \quad p=n^{1 / i}, \quad p=(n / 2)^{1 / i}, \quad p=(n / 3)^{1 / i}, \ldots \\
& \text { and curves } \quad k=p^{i}, \quad k=2 p^{i}, \quad k=3 p^{i}, \ldots \\
& k=n-p^{i}, \quad k=n-2 p^{i}, \quad k=n-3 p^{i}, \ldots .
\end{aligned}
$$

Clearly, a necessary condition for $(p, k) \in E_{i}$ is $p \leq n^{1 / i}$; for example, if $n=600$, $E_{2}$ is included in the vertical strip $\{(p, k): p \leq 23\}, E_{3}$ in $\{(p, k): p \leq 7\}$, etc. In fact, $E_{1}$ and $\bigcup_{i>0} E_{i}$ coincide in the strip $\left\{(p, k): p>n^{1 / 2}\right\}$ : the larger $n$ is, the more $\bigcup_{i>0} E_{i}$ looks like $E_{1}$. This is the reason the pattern for $n=600$ seems to be (and is for $p>23$ ) a union of triangular areas. Figures 2 and 3 show, respectively, $E_{1}$ and $E_{2}$ for very large values of $n$ ( $E_{2}$ is drawn with a dilation along the $p$-axis).

It is easily seen on Figure 1 that:

(1) For $p$ prime, $p>n / 2$, every $\left(\begin{array}{l}n \\ k\end{array}\right)$ satisfying $n-p<k<p$ is divisible by $p$.

(2) For a given $n$, any prime $p$ satisfying $[n / 2]<p \leq n$ is a divisor of $\left(\begin{array}{c}n \\ {[n / 2]}\end{array}\right)$.

(3) For $k>n^{1 / 2}$, prime divisors of $\left(\begin{array}{l}n \\ k\end{array}\right)$ are in the set

$$
\bigcup_{0<j<[n / k]}((n-k) / j, n / j] .
$$

Finally, Figure 4 shows a 3 -dimensional picture of a slice of the set $\{(p, n, k): p$ prime divisor of $\left.\left(\begin{array}{l}n \\ k\end{array}\right)\right\}$.

3. Remarks on the Number of Prime Distinct Divisors of Binomial Coefficients. The problem of a precise estimation for the number $\omega(n, k)$ of prime distinct divisors of $\left(\begin{array}{l}n \\ k\end{array}\right)$ is not solved. It has been studied by Erdős [1], [2] and Erdős 


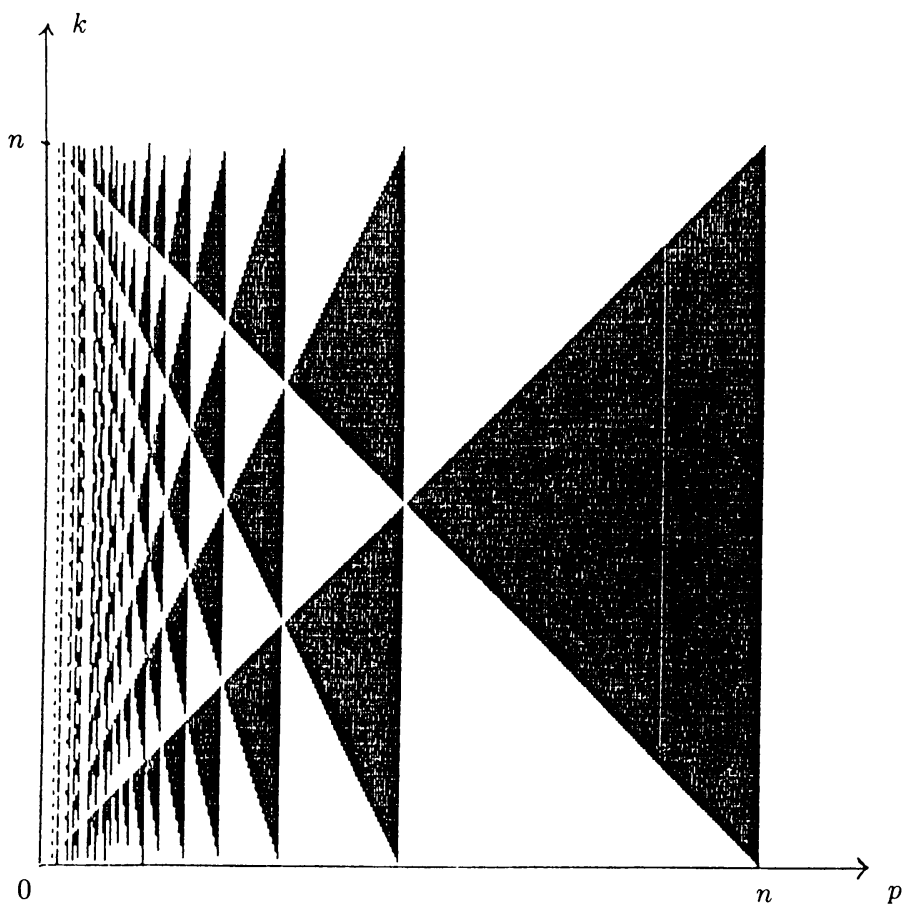

FIGURE 2

The set $E_{1}$ ( $n$ large)

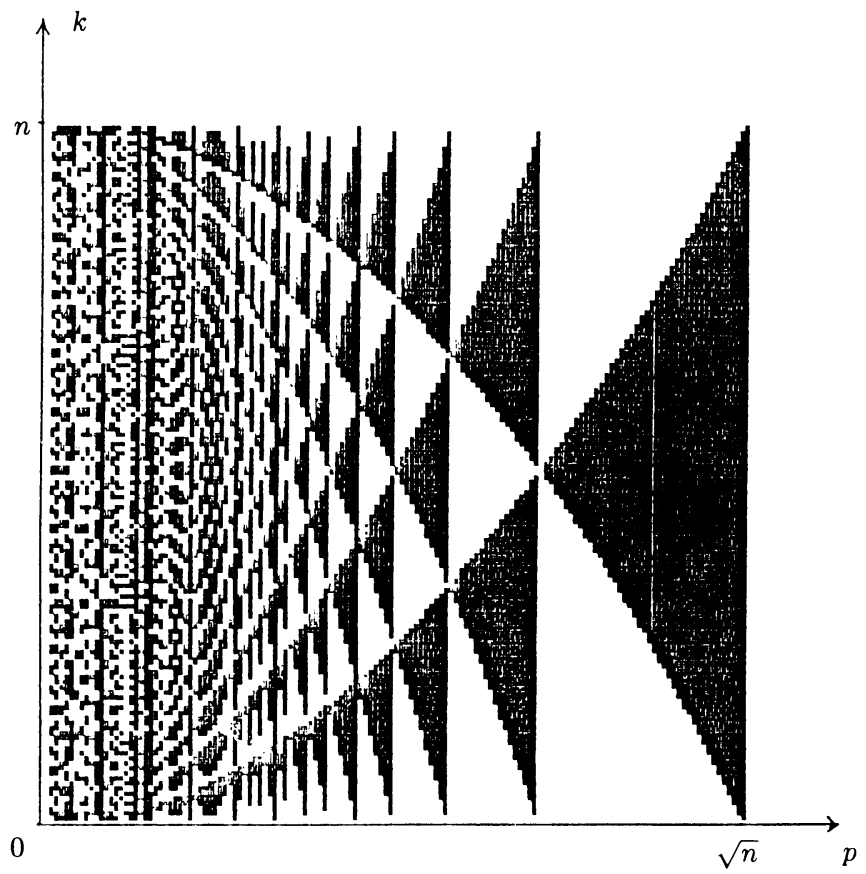

FIGURE 3

The set $E_{2}$ (dilated along p-axis, $n$ large) 


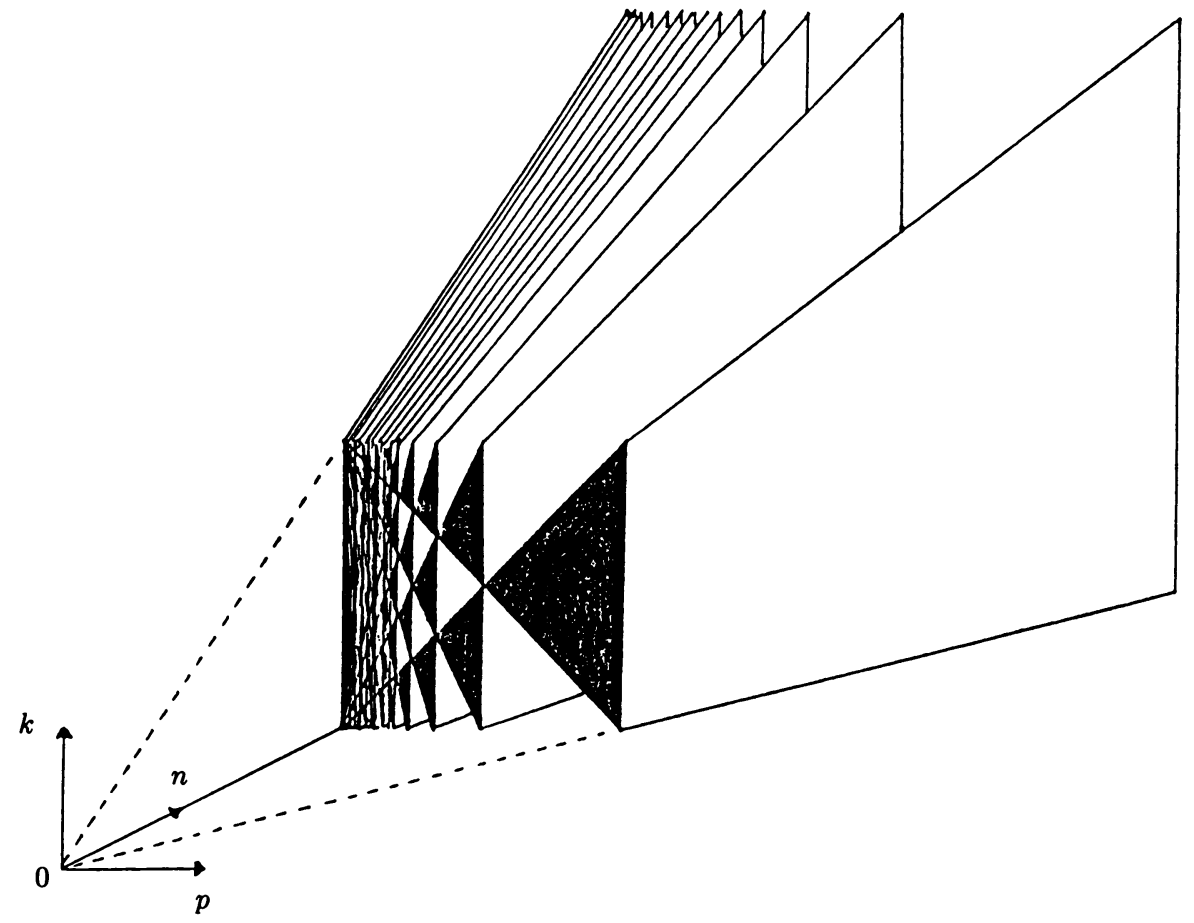

FIGURE 4

A slice of the set of $(p, n, k)$ such that $p$ (prime) divides $\left(\begin{array}{l}n \\ k\end{array}\right)$.

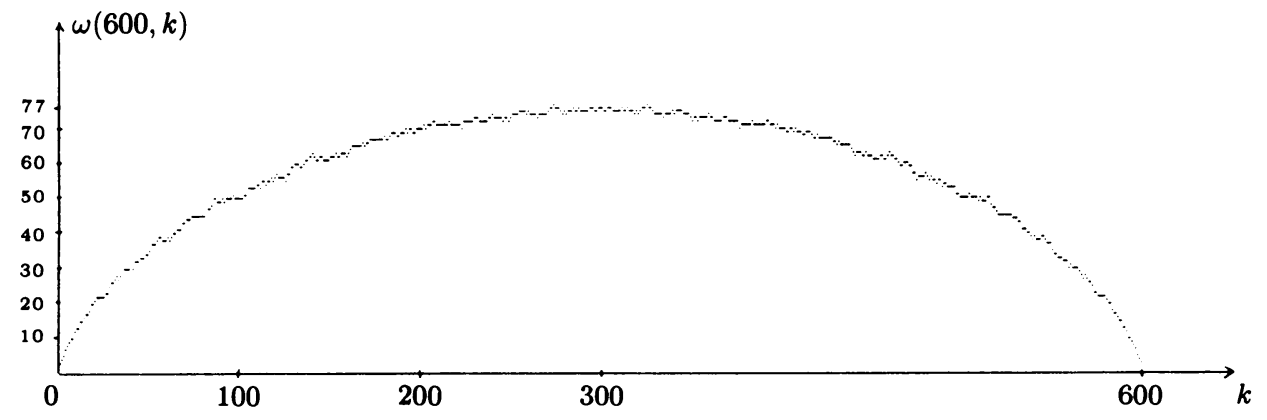

FIGURE 5

The number of prime distinct divisors of $\left(\begin{array}{c}600 \\ k\end{array}\right)$ versus $k$

et al. [3], [4]. Figure 5 shows the graph of the function $k \rightarrow \omega(n, k)$ for $n=600$. We get the same kind of curve for any $n$.

Remark 1. Let $n$ be given.

(a) For $0 \leq k \leq n / 2, k \rightarrow \omega(n, k)$ is roughly an increasing function.

(b) Irregularities have a small range.

This can be seen as a computational illustration of a theorem by Pleasants [6] who proves, among other interesting results, that

(1) There exists a positive function $f$ such that, if $r+f(r)<s \leq n / 2$, then $\omega(n, r) \leq \omega(n, s)$; furthermore, $f(r)=O\left(r /(\log r)^{1 / 2}\right)$. 
(2) There exists a positive function $g$ such that, if $r \leq s \leq n / 2$, then $\omega(n, r)-$ $\omega(n, s) \geq-g(r)$; furthermore, $g(r)=O\left(r /(\log r)^{2}\right)$.

Remark 2. Erdős [1] found the asymptotic formula $\omega(2 n, n) \sim n \log 4 / \log n$. It can be considered as an accurate formula for large values of $n$ as shown below:

$\begin{array}{rrcc}n & \text { Erdős' form } & \omega(2 n, n) & \text { error }(\%) \\ \ldots \ldots \ldots \ldots \ldots \ldots \ldots \ldots \ldots \ldots \ldots \ldots \ldots \ldots \ldots \ldots & \ldots \ldots \ldots \\ 500 & 112 & 116 & 3.45 \\ 1000 & 201 & 208 & 3.37 \\ 2000 & 364 & 373 & 2.41 \\ 5000 & 814 & 837 & 2.75 \\ 15000 & 2163 & 2207 & 1.99\end{array}$

4. On a Conjecture of Erdős. Erdős conjectured that for $n>4,\left(\begin{array}{c}2 n \\ n\end{array}\right)$ is never squarefree. Sárközy [7] proved there exists $n_{0}$ such that the conjecture is true for $n>n_{0}$. Unfortunately, it seems to be very difficult obtaining an effective value for $n_{0}$. Nevertheless, using (C) of Section 1 allows some computation.

Let us note that the problem can be significantly reduced: If $n$ is not a power of 2 , then there are at least two borrows in the subtraction $2 n-n$ in base 2 . Therefore, $\left(\begin{array}{c}2 n \\ n\end{array}\right)$ is divisible by 4 .

When $n=2^{a}$ one can try testing the divisibility of $\left(\begin{array}{c}2 n \\ n\end{array}\right)$ by 9 . A sufficient condition for the divisibility is that the expansion of $n$ in base 3 has at least two digits 2: in this case there are at least two borrows in the subtraction $2 n-n$ in base 3. The test performed only on the 256 last digits of $n$ in base 3 , for $n=2^{a}$, $3 \leq a \leq 42205184$, showed that the sufficient condition is satisfied except for

$$
\begin{array}{ll}
a=4,5,24 ; & \text { however in these cases } 9 \text { divides }\left(\begin{array}{c}
2 n \\
n
\end{array}\right) ; \\
a=6 ; & \text { in this case } 25 \text { divides }\left(\begin{array}{c}
128 \\
64
\end{array}\right) ; \\
a=8 ; & \text { in this case } 49 \text { divides }\left(\begin{array}{c}
512 \\
256
\end{array}\right) .
\end{array}
$$

Thus, the Erdös conjecture is true if

(1) $4<n \leq 2^{42205184}$,

(2) $n \neq 2^{a}$.

Université de Paris-sud

Mathématiques, Bât. 425

91405 Orsay Cedex, France

1. P. ERDős, "Über die Anzahl der Primfaktoren von $\left(\begin{array}{l}n \\ k\end{array}\right)$," Arck. Math., v. 24, 1973, pp. 53-56.

2. P. ERDős, "Some unconventional problems in number theory," Acta Math. Acad. Sci. Hungar., v. 33, 1979, pp. 71-80.

3. P. Erdős, R. L. Graham, I. RUzSA \& E. G. Straus, "On the prime factors of $\left(\begin{array}{c}2 n \\ n\end{array}\right)$," Math. Comp., v. 29, 1975, pp. 83-92.

4. P. ERDős, H. Gupta \& S. P. KhaRe, "On the number of distinct prime divisors of $\left(\begin{array}{l}n \\ k\end{array}\right)$," Utilitas Math., v. 10, 1976, pp. 51-60.

5. P. Goetgheluck, "Computing binomial coefficients," Amer. Math. Monthly, v. 94, 1987, pp. $360-365$.

6. P. A. B. Pleasants, "The number of prime factors of binomial coefficients," J. Number Theory, v. 15, 1982, pp. 203-225.

7. A. SÁRKÖzY, "On divisors of binomial coefficients, I," J. Number Theory, v. 20, 1985, pp. 70-80. 